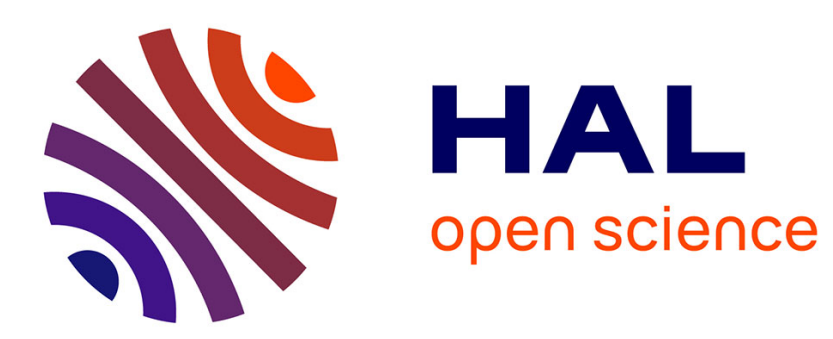

\title{
Hydrodynamic attraction of immobile particles due to interfacial forces
}

\author{
Julien Morthomas, Alois Würger
}

\section{To cite this version:}

Julien Morthomas, Alois Würger. Hydrodynamic attraction of immobile particles due to interfacial forces. Physical Review E: Statistical, Nonlinear, and Soft Matter Physics, 2010, 81, pp.051405. 10.1103/PhysRevE.81.051405 . hal-00486901

\section{HAL Id: hal-00486901 https://hal.science/hal-00486901}

Submitted on 27 May 2010

HAL is a multi-disciplinary open access archive for the deposit and dissemination of scientific research documents, whether they are published or not. The documents may come from teaching and research institutions in France or abroad, or from public or private research centers.
L'archive ouverte pluridisciplinaire HAL, est destinée au dépôt et à la diffusion de documents scientifiques de niveau recherche, publiés ou non, émanant des établissements d'enseignement et de recherche français ou étrangers, des laboratoires publics ou privés. 


\title{
Hydrodynamic attraction of immobile particles due to interfacial forces
}

\author{
Julien Morthomas and Alois Würger \\ CPMOH, Université Bordeaux 1 \& CNRS, \\ 351 cours de la Libération, 33405 Talence, France
}

\begin{abstract}
Applying the method of reflections, we derive the flow pattern around a confined colloidal particle with quasislip conditions at its surface, in powers of the ratio $a / h$ of particle radius and wall distance. The lowest order corresponds to a single reflection at the confining wall. Significant corrections occur at higher order: the linear term in $a / h$ modifies the amplitudes of the well-known one-reflection approximation, whereas new features arise in quadratic order. Our results agree with recent experiments where thermoosmosis drives hydrodynamic attractive forces in confined colloids.
\end{abstract}

PACS numbers: $66.10 . \mathrm{C}, 82.70 .-\mathrm{y}, 47.57 . J-$ 


\section{INTRODUCTION}

Colloidal transport and small-scale flow in complex fluids are mostly driven by interfacial forces, that may arise from an electric field, or thermal or chemical gradients [1]. These external fields induce a quasi-slip velocity at the particle surface and a corresponding fluid flow. For example, salinity and temperature inhomogeneities have been used for focussing and spreading colloidal particles in a microchannel, or for locally augmenting their density by several orders of magnitude [2-4]. Confinement perpendicular to the applied field modifies both the fluid velocity field and the colloid kinetics. As a particle approaches a solid boundary, its sedimentation slows down, because of an additional velocity component proportional to the the ratio $a / h$ of the particle size $a$ and the distance $h$; on the contrary, the electrophoretic mobility of a particle approaching a wall varies as $(a / h)^{3}[5-9]$. The steady state is reached if the viscous stress and the repulsion by the wall counterbalance the interfacial driving force; then the particle is immobile, and the surrounding velocity field is squeezed by the boundary. Since the particle continues to move the fluid along its surface, it creates a flow with incoming radial and outgoing vertical components.

As a striking consequence, a second nearby particle experiences an effective attractive force. Cluster formation of charged micron-sized colloids has been observed during electrophoretic deposition and in an ac field [10-15]. The crucial role of the electroosmotic flow is confirmed by the fact that aggregation is reversible, i.e., the ordering disappears after switching off the external field. In the case of electroosmosis in a DC field, the flow $u_{0}=\mu E$ varies linearly with the external field $E$ [1] and leads to particle clustering at the electrode $[11,15]$.

More recently, several studies investigated confined colloids in a thermal gradient $[16,17]$. Besides the reversible aggregation, these experiments recorded the kinetics of small tracer particles and reported how the effective attraction of neighbor particles varies with their distance. Thermal forces have been observed for charged colloidal particles close to a glass plate with a vertical temperature gradient $[16,17]$. The thermoosmotic flow along the particle surface $[18,19]$ induces a radial inward flow, which results in hydrodynamic attraction along the confining wall and favors cluster formation. Thermoosmotic driving accounts both for the double-layer enthalpy transport and the thermoelectric response of the salt solution, and thus may take both signs, depending on the solute and solvent properties [20]. 
In the present paper we study the flow pattern induced by a constant electric field or a temperature gradient. The velocity field and the resulting hydrodynamic interactions are determined as series in powers of the parameter

$$
\varepsilon=\frac{a}{h} \text {. }
$$

We consider a spherical particle at rest at a distance $h$ from a solid boundary plane $B$. The stationary flow $\mathbf{u}(\mathbf{r})$ is described by Stokes' equation for an incompressible fluid of viscosity $\eta$

$$
\eta \nabla^{2} \mathbf{u}=\nabla P, \quad \nabla \cdot \mathbf{u}=0
$$

Stick boundary conditions apply at the confining wall $B$, whereas the osmotic flow at the particle surface $S$ imposes a quasi-slip velocity,

$$
\left.\mathbf{u}\right|_{B}=0,\left.\quad \mathbf{u}\right|_{S}=-u_{S} \mathbf{e}_{\theta}
$$

The unit vector parallel to the particle surface, $\mathbf{e}_{\theta}$, is directed downwards; thus $u_{S}>0$ corresponds to an upward boundary flow in Fig. 1. For a spherical particle with a homogeneous surface suspended in a bulk phase the boundary velocity varies with the sine of the polar angle [1],

$$
u_{S}=u_{0} \sin \theta
$$

In the confined geometry studied here, this relation remains valid if the external field is not too strongly deformed by the presence of the particle and the solid boundary. A more general form occurs if the electric permittivities or thermal conductivities show strong discontinuities at the solid-fluid interfaces.

In Sect. 2 we introduce the velocity field in spherical coordinates and discuss the simple example of an immobile particle without confinement. Sect. 3 presents the method of reflections and the systematic expansion in powers of $\varepsilon$, and gives explicit results for the radial velocity component. In Sect. 4 we discuss recent experiments where colloidal particles are confined through thermoosmotic driving. In Sect. 5 we abondon the particular form (3) for the quasislip velocity and study the general case.

\section{FLUID FLOW WITHOUT CONFINEMENT}

We start with the case of an unconfined immobile particle, which can be realized by counterbalancing the osmotic surface force by an external body force, such as optical tweezers. 


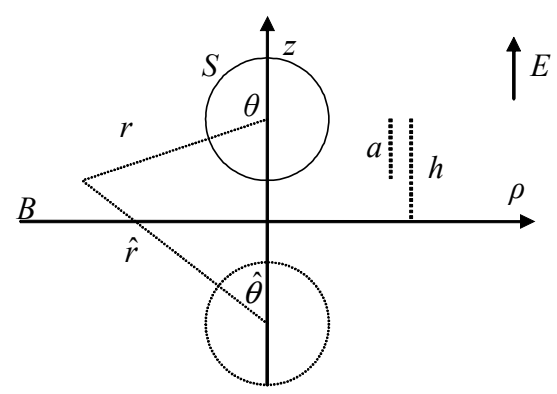

FIG. 1: Confined particle $S$ of radius $a$ at a distance $h$ from the boundary $B$. We indicate spherical coordinates $r, \theta$ with respect to the particle, $\hat{r}, \hat{\theta}$ with respect to the image particle, and cylindrical coordonnates $\rho, z$.

Then the general solution of Stokes' equation in spherical coordinates reads

$$
\mathbf{v}(r, \theta)=u_{0} \sum_{n=1}^{\infty} \frac{a^{n}}{r^{n}}\left(R_{n}(\theta) \mathbf{e}_{r}+T_{n}(\theta) \mathbf{e}_{\theta}\right) .
$$

(A corresponding expansion for the stream function has been given by Brenner [5].) Writing the vector Laplace and the divergence operators in the local basis $\left\{\mathbf{e}_{r}, \mathbf{e}_{\theta}\right\}$, one finds the radial and tangential components in terms of Legendre polynomials and their derivatives,

$$
\begin{gathered}
R_{n}=p_{n} P_{n}(\cos \theta)+q_{n} P_{n-2}(\cos \theta), \\
T_{n}=-\frac{p_{n}(n-2)}{n(n+1)} \frac{d P_{n}}{d \theta}-\frac{q_{n}}{n-1} \frac{d P_{n-2}}{d \theta} .
\end{gathered}
$$

The solution of Eq. (1) consists of inhomogeneous and homogeneous contributions with coefficients $p_{n}$ and $q_{n}$, respectively. The former are related to a non-uniform pressure proportional to $\frac{4 n-2}{n+1} p_{n} P_{n} / r^{n+1}$. Homogeneous solutions exist for $n \geq 3$; this implies $q_{1}=0=q_{2}$.

With the quasi-slip boundary condition at the particle surface $S$ one has

$$
p_{1}=1, \quad q_{3}=-1
$$

The radial and tangential compenents of the velocity field are given by

$$
\frac{v_{r}}{u_{0}}=\cos \theta\left(\frac{a}{r}-\frac{a^{3}}{r^{3}}\right), \frac{v_{\theta}}{u_{0}}=-\frac{\sin \theta}{2}\left(\frac{a}{r}+\frac{a^{3}}{r^{3}}\right) .
$$

In terms of hydrodynamic multipoles [21], this flow consists of a "stokeslet" of strength $p_{1}$ and a "source doublet" $q_{3}$. The latter arises from the interfacial forces and varies as $1 / r^{3}[1]$, whereas the external body force results in the stokeslet with the characteristic dependence 
on $1 / r$. Thus the flow pattern in the vicinity of an immobile particle, as given by Eq. (7) and illustrated in Fig. 2a, differs significantly from that of a particle moving at velocity $u_{0}$ due to phoresis.

\section{CONFINEMENT AND IMAGE FLOW}

\section{A. Velocity field due to an image particle}

Stick boundary conditions at the confining wall require both radial and vertical velocity components to vanish on $B$. Since this is not possible with the vector field $\mathbf{v}$, we add a second solution $\hat{\mathbf{v}}$ of Stokes' equation, such that the sum

$$
\mathbf{u}=\mathbf{v}+\hat{\mathbf{v}}
$$

satisfies both conditions in Eq. (2). The flow $\hat{\mathbf{v}}$ may be viewed in terms of an image particle at $z=-h$. Accordingly, we expand $\hat{\mathbf{v}}(\hat{r}, \hat{\theta})$ in a series identical to Eq. (4), yet in powers of the distance $\hat{r}$ defined in Fig. 1 ; the radial and tangential compenents $\hat{R}_{n} \hat{\mathbf{e}}_{r}+\hat{T}_{n} \hat{\mathbf{e}}_{\theta}$ depend on the angle $\hat{\theta}$ with coefficients $\hat{q}_{n}, \hat{p}_{n}$.

The boundary condition at the confining wall imposes $\left.(\hat{\mathbf{v}}+\mathbf{v})\right|_{B}=0$. Separating the parallel and perpendicular velocity components $u_{\rho}$ and $u_{z}$, expanding the Legendre polynomials in powers of their argument, and using $\hat{r}=r$ and $\theta+\hat{\theta}=\pi$, one finds a set of equations relating $\hat{q}_{m}, \hat{p}_{m}$ to $q_{n}, p_{n}$. For later use we give their solution up to $n=4$,

$$
\begin{aligned}
& \hat{p}_{1}=-p_{1}, \quad \hat{p}_{3}=\frac{24}{5 \varepsilon} p_{2}-\frac{24}{5} q_{3}+\frac{11}{5} p_{3}, \\
& \hat{p}_{2}=-\frac{2}{\varepsilon} p_{1}-p_{2}, \quad \hat{p}_{4}=-\frac{50}{7 \varepsilon} p_{3}+\frac{40}{7} q_{4}-\frac{23}{7} p_{4}, \\
& \hat{q}_{3}=\frac{2}{\varepsilon^{2}} p_{1}+\frac{16}{5 \varepsilon} p_{2}-\frac{11}{5} q_{3}+\frac{4}{5} p_{3}, \quad \hat{q}_{5}=-\frac{8}{\varepsilon} q_{4}, \\
& \hat{q}_{4}=-\frac{6}{\varepsilon^{2}} p_{2}+\frac{6}{\varepsilon} q_{3}-\frac{48}{7 \varepsilon} p_{3}+\frac{23}{7} q_{4}-\frac{12}{7} p_{4} .
\end{aligned}
$$

Thus we have expressed $\mathbf{u}$ in terms of $q_{n}$ and $p_{n}$.

\section{B. Perturbation expansion}

The coefficients $q_{n}$ and $p_{n}$ have to be determined from the boundary condition at the particle surface $S$. We propose an iteration scheme based on the method of reflections [6] 


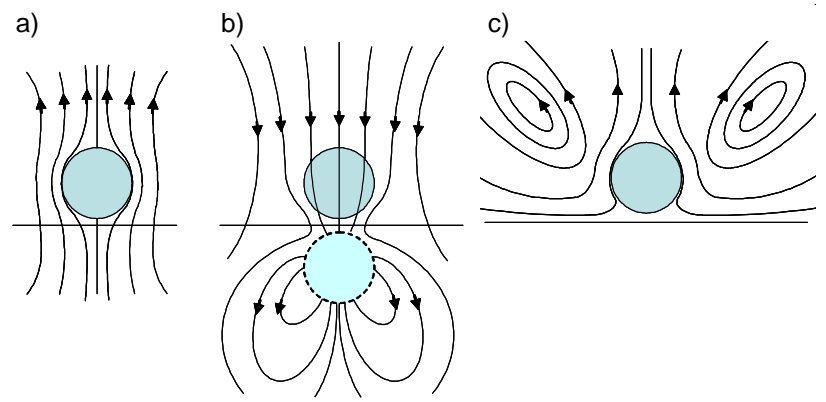

FIG. 2: a) Velocity field $\mathbf{v}^{(0)}$ of an immobile particle at $z=h$, as given in Eq. (7). b) Velocity field $\hat{\mathbf{v}}^{(0)}$ due to the image particle at $z=-h$. c) The superposition $\mathbf{u}^{(0)}$ satisfies the stick boundary condition at the wall $B$.

and resulting in a convergent sequence $\mathbf{u}^{(k)}=\mathbf{v}^{(k)}+\hat{\mathbf{v}}^{(k)}$. At each order the image flow $\hat{\mathbf{v}}^{(k)}$ is determined from $\mathbf{v}^{(k)}$ according to

$$
\left.\left(\hat{\mathbf{v}}^{(k)}+\mathbf{v}^{(k)}\right)\right|_{B}=0
$$

the coefficients $\hat{q}_{n}^{(k)}$ and $\hat{p}_{n}^{(k)}$ are obtained from $q_{n}^{(k)}$ and $p_{n}^{(k)}$ by using Eq. (10).

In the iteration step we calculate the flow $\mathbf{v}^{(k+1)}$ from $\hat{\mathbf{v}}^{(k)}$ and the boundary condition at $S$. In order to keep the problem of finite dimension, we use $\varepsilon$ as a book-keeping parameter and evaluate the boundary condition to accuracy $\varepsilon^{k+1}$, that is

$$
\left.\left(\mathbf{v}^{(k+1)}+\hat{\mathbf{v}}^{(k)}\right)\right|_{S}=O\left(\varepsilon^{k+2}\right) .
$$

Thus we obtain the coefficients $q_{n}$ and $p_{n}$ as series in $\varepsilon$. Eqs. (11) and (12) may be considered as reflections of the hydrodynamic flow at the confining wall and the particle surface [6]; successive application gives the velocity field to any desired precision.

As starting point we take the flow around an immobile particle without confinement, as given in Eq. (7). The image flow $\hat{\mathbf{v}}^{(0)}$ follows from Eq. (10) and is illustrated in panel b of Fig. 2. Besides the stokeslet $\hat{p}_{1}$ and the source doublet $\hat{q}_{3}$, it comprises additional terms $\hat{p}_{2}, \hat{p}_{3}, \hat{q}_{4}$ corresponding to a "Stokes doublet". As illustrated in Fig. 2, the image flow differs qualitatively from $\mathbf{v}^{(0)}$. By construction, both parallel and perpendicular components of $\mathbf{u}^{(0)}$ vanish at the boundery $B$; on the particle surface the error is of the order $\varepsilon u_{0}$. 


\section{Radial velocity}

The above coefficients provide an explicit expression for the velocity field $\mathbf{u}^{(0)}$. The flow parallel to the confining wall is of particular interest for experiment; we thus evaluate its radial component in cylindrical coordinates in the plane $z=h$ as a function of $\rho$,

$$
\frac{u_{\rho}^{(0)}(\rho, h)}{u_{0}}=-6 \frac{\rho a h\left(a^{2}+h^{2}\right)}{\hat{r}_{h}^{5}}+60 \frac{\rho a^{3} h^{3}}{\hat{r}_{h}^{7}} .
$$

Note that both terms are in inverse powers of $\hat{r}_{h}=\sqrt{\rho^{2}+4 h^{2}}$ and thus stem from $\hat{\mathbf{v}}^{(0)}$; the radial compenent of $\mathbf{v}^{(0)}$ vanishes at $z=h$. Putting $a=h$ we recover the result given previously in [17]. The two terms of opposite sign result in the minimum at $\rho \approx 2 a$. Since the exact radial velocity vanishes at the particle surface, the finite value at $\rho=a$ reflects the approximate treatment of the boundary condition (12).

Corrections are calculated from the above iteration scheme. After eliminating $\hat{r}$ and $\hat{\theta}$ through the relations $\hat{r} \cos \hat{\theta}=a \cos \theta+2 h$ and $\hat{r} \sin \hat{\theta}=a \sin \theta$ on $B$, and writing both sides as series in $\cos \theta$, we obtain a system of equations for the $q_{n}, p_{n}$, which can be solved explicitly after expanding in powers of $\varepsilon$. The first correction reads

$$
\frac{u_{\rho}^{(1)}}{u_{0}}=6 \frac{\rho a h^{3}\left(\varepsilon^{2} q_{3}-p_{1}\right)}{\hat{r}_{h}^{5}}-60 q_{3} \frac{\rho a^{3} h^{3}}{\hat{r}_{h}^{7}},
$$

with modified coefficients

$$
p_{1}=1+\frac{9}{8} \varepsilon, \quad q_{3}=-1-\frac{3}{8} \varepsilon
$$

(We suppress the label $k$ in $p_{n}$ and $q_{n}$.) As shown in Fig. 3, the linear correction enhances the radial velocity by more than a factor 2 .

At second-order $k=2$, the corrections to $p_{1}$ and $q_{3}$ read $\frac{81}{64} \varepsilon^{2}$ and $-\frac{27}{64} \varepsilon^{2}$, respectively, and two new coefficients $p_{2}=-\frac{15}{16} \varepsilon^{2}$ and $q_{4}=\frac{9}{16} \varepsilon^{2}$ appear. Inserting the image coefficients (10) and using the function (14), we have

$$
\frac{u_{\rho}^{(2)}}{u_{0}}=\frac{u_{\rho}^{(1)}}{u_{0}}+\frac{p_{2}}{2}\left(\frac{\rho a^{2}}{\hat{r}_{h}^{3}}-\frac{a^{2}}{\rho^{2}}\right)+\ldots
$$

The expression in brackets vanishes at large distances; the terms $p_{2}$ and $q_{4}$ mainly affect the fluid velocity close to the particle. According to Fig. 3, the minimum is shifted to a slightly larger distance, and the behavior close to the particle is improved in view of the boundary condition $u_{\rho}(a, h)=0$. 


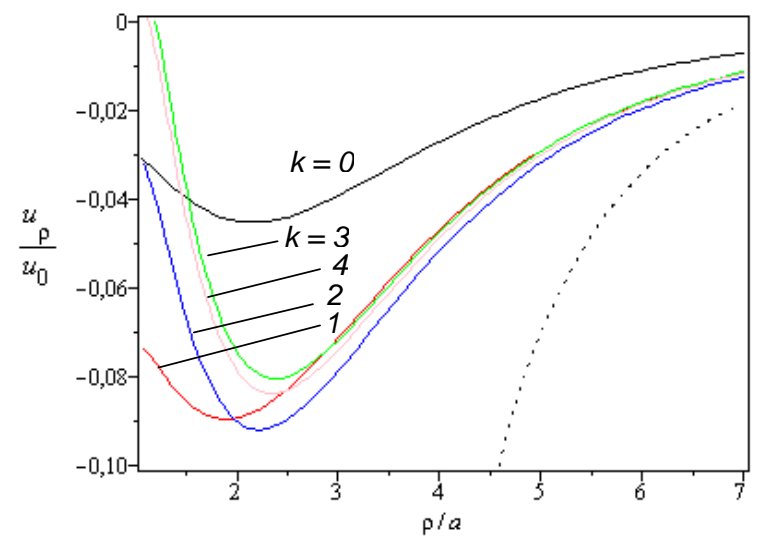

FIG. 3: Radial velocity $u_{\rho}^{(k)}$ in the plane $z=h$ as a function of $\rho$ for the aspect ratio $\varepsilon=\frac{2}{3}$; we give the results of $k=0, \ldots, 4$ reflections at the particle surface. The dotted line gives the power law $1 / \rho^{4}$ of Eq. (16).

Reflections of higher order 3 and 4 do not significantly modify the picture; Fig. 3 suggests a rapid convergence. Each subsequent reflection at $S$ adds a factor $\varepsilon$; the new contributions to the component $\mathbf{v}^{(k)}$ are of the form $p_{k} \sim \varepsilon^{k}$ and $q_{k+2} \sim \varepsilon^{k}$. To fourth order, the two first coefficients read explicitly

$$
\begin{aligned}
& p_{1}=1+\frac{9}{8} \varepsilon+\frac{81}{64} \varepsilon^{2}+\frac{217}{512} \varepsilon^{3}+\frac{1809}{4096} \varepsilon^{4}+\ldots \\
& a_{3}=-1-\frac{3}{8} \varepsilon-\frac{27}{64} \varepsilon^{2}-\frac{191}{2560} \varepsilon^{3}-\frac{1479}{20480} \varepsilon^{4}+\ldots
\end{aligned}
$$

The numerical factors suggest that subsequent coefficients of odd and even order in $\varepsilon$ are of comparable size, e.g., $\frac{9}{8} \sim \frac{81}{64}$ and $\frac{217}{512} \sim \frac{1809}{4096}$ in $p_{1}$; the same pattern occurs for the other coefficients. The above numbers and our partial results for higher orders $k \geq 5$ suggest that these pairs converge rather rapidly. Moreover we note that some image coefficients $\hat{q}_{n}$, $\hat{p}_{n}$ diverge in the limit of small $\varepsilon$, such as $\hat{p}_{2} \sim-2 / \varepsilon$, the corresponding velocity field is nonetheless well behaved in the upper half space.

The zero-order expression $\mathbf{u}^{(0)}$ becomes exact in the limit $\varepsilon \rightarrow 0$, but provides a rather poor approximation for real systems where the parameter $\varepsilon$ is not small. For example, the distance of charged colloids, $h \approx a+\lambda$, exceeds the radius $a$ by about one Debye length $\lambda$; for micron-size particles the ratio $a / h$ is rather close to unity.

At large distances $(\rho \gg h$ or $z \gg h)$ the velocity shows power laws as a function of the 
radial or vertical coordinates. Expanding the radial component in powers of $1 / \rho$ we find

$$
\frac{u_{\rho}}{u_{0}}=-\beta \frac{a h^{2} z}{\rho^{4}}+\ldots
$$

with the numerical coefficient $\beta=6\left(1+\frac{9}{8} \varepsilon+\ldots\right)$. The vertical component vanishes even more rapidly, $u_{z} / u_{0}=9 a h^{2} z^{2} / \rho^{5}$ to leading order in $\varepsilon$ and $1 / \rho$. On the vertical axis $(\rho=0)$ we find in powers of $1 / z$

$$
\frac{u_{z}}{u_{0}}=\beta \frac{h^{2} a}{z^{3}}+\ldots
$$

\section{HYDRODYNAMIC INTERACTIONS}

\section{A. Lateral attraction}

The radial velocity field advects the fluid along the solid boundary $B$ and acts as an effective force on nearby particles. Their current $J=-D \partial_{\rho} n+n U$ comprises diffusion with the Einstein coefficient $D$ and the advection velocity $U(\rho)=u_{\rho}(\rho, h)$. The steady-state probability distribution function $n(\rho)=n_{0} e^{-V / k_{B} T}$ is readily integrated, with a pseudopotential $V$. For two beads of equal size, the mutual forces lead to a factor of 2. Fig. 3 shows that $k=1$ provides a good approximation at distances $\rho>2 a$. Then the effective pair potential reads as

$$
\frac{V}{k_{B} T}=8 \frac{u_{0} a}{D}\left(\frac{h^{3}\left(\varepsilon^{2} q_{3}-p_{1}\right)}{\hat{r}_{h}^{3}}-6 q_{3} \frac{a^{2} h^{3}}{\hat{r}_{h}^{5}}\right)
$$

the force $F(\rho)=-\partial_{\rho} V$ is required to keep the particles at a distance $\rho$. The dimensionless Péclet number $\mathrm{Pe}=u_{0} a / D$ compares the advection velocity $u_{0}$ with diffusion over a particle size; if $\mathrm{Pe} \gg 1$, hydrodynamic interactions provide an efficient trap.

The pair correlation function and the pseudopotential $V$ of polystyrene beads in water have been determined by single-particle tracking [16]; a direct measurement of the force $F$ has been reported for silica beads in a water/alcohol mixture [17]. In Fig. 4 we compare these experiments with our result for $k=1$, as given in (18). The data of [16] are fitted with $\mathrm{Pe}=6$; with $D=k_{B} T / 6 \pi \eta a$ and $a=1 \mu \mathrm{m}$ this corresponds to a slip velocity $u_{0}=1.3 \mu \mathrm{m} / \mathrm{s}$; with the value $\nabla T=0.28 \mathrm{~K} / \mu \mathrm{m}$ given in [16], the transport coefficient $D_{T} \sim 5 \mu \mathrm{m}^{2} / \mathrm{Ks}$ lies in the range expected for weak electrolytes [20]. Thus the first-order approximation of Eqs. (14) and (18) provides a quantitive description for the flow around confined colloids. The fit of the force measurements of Ref. [17] lead to a four times larger Péclet number $\mathrm{Pe}=24$ 


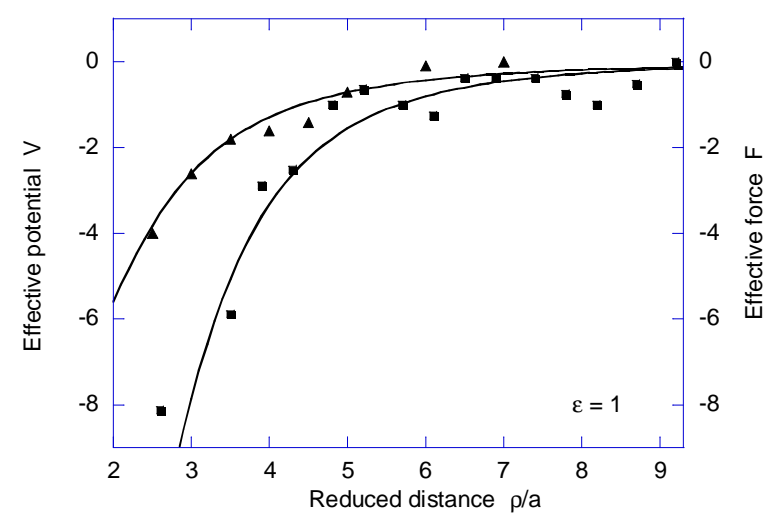

FIG. 4: Comparison with experimental data. Triangles give measured values for effective potential $V$ in units of $k_{B} T$ [16]. Data for the hydrodynamic force $F=-\partial_{\rho} V$ [17] are indicated by squares and given in units of $k_{B} T / \mu \mathrm{m}$. The curves are calculated from (18) and $F=-\partial_{\rho} V$ with $\mathrm{Pe}=6$ and 24 , respectively.

and a slip velocity of about $10 \mu \mathrm{m} / \mathrm{s}$. From Fig. 3 it is clear that corrections of higher order $(k>1)$ would hardly modify the theroetical curve; yet note that the usual zero-order approximation $k=0$ underestimates the attractive force roughly by a factor of two.

\section{B. Particle rotation}

Finally we discuss the curl of the velocity field $\mathbf{u}$ that rotates a neighbor particle, as would do an applied torque. As a consequenc, two nearby beads turn in the $\rho$ - $z$-plane about their axes at a frequency $\Omega$, as shown in Fig. 5a. Evaluating $\boldsymbol{\nabla} \times \mathbf{u}$ to leading order in $\varepsilon$ and $1 / r_{h}$ one finds

$$
\Omega=6 u_{0} h^{2} a \rho / \hat{r}_{h}^{5}
$$

For nearby particles one has $\Omega \sim u_{0} / a$. Rotation at frequency of about $1 \mathrm{~Hz}$ has been observed experimentally for polystyrene beads in an ac electric field [23]. With the above parameters, similar values are expected to occur for thermo-osmotic flow. 
a)

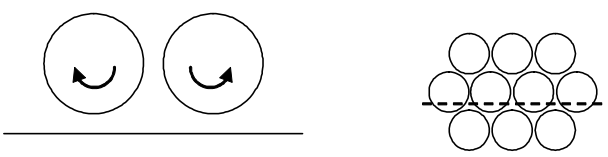

b)



FIG. 5: a) Particle rotation due to the curl of the neighbor velocity field. b) In aggregates, only the outer particles rotate significantly. Between inner particles, the vertical fluid flow in the "chimneys" is indicated along the dashed line.

\section{Cluster size}

At sufficiently high particle density, the hydrodynamic attraction favors the formation of cluster at the confining plate; small two-dimensional crystals have indeed observed, both in an external electric field $\mathbf{E}[14,15]$ and a temperature gradient $\boldsymbol{\nabla} T[16,17]$. There is hardly any radial flow towards the inner particles of such a cluster; thus the osmotic flow at the particle surface is counterbalanced by a downward current in the chimneys between particles, as shown schematically in Fig. 5b. How this flow pattern influences the size, shape, and stability of the clusters, remains an open question.

\section{DISTORTION OF THE DRIVING FIELD}

Throughout this paper we have used the quasislip condition on the particle surface given in Eq. (2). If this form is generally valid for a spherical particle in a bulk liquid, it does not necessarily apply to the case of a particle close to a solid boundary. Here we discuss in detail the case of a temperature gradient, where the validity (2) depends on the values of the thermal conductivities of the particle, the liquid, and the solid boundary, which we denote $\kappa_{P}, \kappa_{L}$, and $\kappa_{B}$, respectively. The quasislip velocity

$$
u_{S}=-D_{T} \nabla_{\|} T
$$

is given by the tangential component of the local temperature gradient at the particle surface.

We start from the applied temperature $T_{\text {ext }}=T_{0}+z \nabla T$ with a constant gradient $\nabla T$ along the $z$-axis. A more complex field $T(\mathbf{r})$ arises in the vicinity of a colloidal particle. It 
satisfies the stationary diffusion equation

$$
\nabla^{2} T=0
$$

both the temperature and the normal heat flux are continuous at the interfaces,

$$
T_{i}=T_{o}, \quad\left(\kappa_{i} \nabla T_{i}-\kappa_{o} \boldsymbol{\nabla} T_{o}\right) \cdot \mathbf{n}=0
$$

where $i, o$ indicate the inner and outer boundary values, and $\mathbf{n}$ is the normal vector. In the following we derive the local gradient as a power series in $\varepsilon$, and how it varies along the particle surface.

First consider a spherical bead in an infinite liquid phase. Solving (21) and (22) inside and outside the bead and matching the solutions at the interface, one readily finds the dipolar deformation

$$
T=T_{0}+\nabla T\left(r+\alpha_{1} \frac{a^{3}}{r^{2}}\right) \cos \theta
$$

for further use we note the well-known contrast factors at spherical and planar surfaces,

$$
\alpha_{n}=\frac{\kappa_{L}-\kappa_{P}}{\left(1+\frac{1}{n}\right) \kappa_{L}+\kappa_{P}}, \quad \gamma=\frac{\kappa_{L}-\kappa_{B}}{\kappa_{L}+\kappa_{B}} .
$$

If the liquid is a much better heat conductor than the particle, the gradient at the surface $r=a$ is enhanced by a factor $\frac{3}{2}$; in the opposite case it vanishes as $\kappa_{L} / \kappa_{P}$.

Now we add the solid boundary $B$. A rapidly convergent expression is obtained by writing the temperature in the liquid phase as the sum of two series, located at the positions of the particle $z=h$ and of its image $z=-h$,

$$
\delta T_{L}=a \nabla T \sum_{n \geq 0}\left(c_{n} \frac{a^{n+1}}{r^{n+1}} P_{n}(\cos \theta)+\hat{c}_{n} \frac{a^{n+1}}{\hat{r}^{n+1}} P_{n}(\cos \hat{\theta})\right),
$$

whereas that in the solid phase consists of a similar series located at the particle position,

$$
\delta T_{B}=a \nabla T \sum_{n \geq 0} d_{n} \frac{a^{n+1}}{r^{n+1}} P_{n}(\cos \theta) .
$$

The coordinates are defined in Fig. 1. The coefficients $c_{n}, \hat{c}_{n}, d_{n}$ are determined perturbatively, very much like in Sect. 3 .

We start from the solution in the absence of the solid boundary, as given in (23) with the only coefficient $c_{1}^{(0)}=\alpha$. Continuity of temperature and heat flux at the boundary $S$ is readily imposed and lead to

$$
c_{1}^{(0)}=\alpha_{1}, \quad \hat{c}_{1}^{(0)}=\alpha_{1} \gamma, \quad d_{1}^{(0)}=\alpha_{1} \gamma \varepsilon^{2} .
$$


The iteration step consists in determining the coefficients in next order through the boundary conditions at the particle surface, with the temperature field inside $\delta T_{P}=$ $a \nabla T \sum_{n} e_{n}(r / a)^{n} P_{n}$. We start from the field proportional to $\hat{c}_{1}^{(0)}$ and find explicit expressions for two boundary conditions. Here we give the relevant coefficient $(n \geq 1)$,

$$
c_{n}^{(1)}=(-1)^{n} \frac{n+1}{2^{n+2}} \alpha_{n} \varepsilon^{n+2} \hat{c}_{1}^{(0)} .
$$

The tangential component of the temperature gradient at the particle surface is obtained from $(25)$ as the derivative $\nabla_{\|} T=(1 / a) d T_{L} / d \theta$ at $r=a$. Expanding $P_{k}(\cos \hat{\theta})$ in powers of $\cos \theta$ and rearranging the terms, we find

$$
\begin{aligned}
\nabla_{\|} T= & \sin \theta \nabla T\left(1+\alpha_{1}\right) \\
& +\sin \theta \nabla T \sum_{n}\left(1+\alpha_{n}\right) \frac{d P_{n}(x)}{d x} c_{n}^{(1)} .
\end{aligned}
$$

The first term on the right-hand side gives the local gradient in the absence of the solid boundary. The second one arises from the discontinuity of the heat conductivity at $B$; accordingly, it vanishes for $\kappa_{L}=\kappa_{B}$, that is, for $\gamma=0$.

Thus the boundary velocity in the absence of $B$ takes the well-known form

$$
u_{0}=\frac{2 \kappa_{L}}{2 \kappa_{L}+\kappa_{P}} D_{T} \nabla T
$$

which is the basis of (3). The right-hand side of Eq. (28) gives the change due to the presence of the solid boundary. The relation (20) implies a corresponding modification of the boundary velocity,

$$
\frac{u_{S}}{u_{0}}=\left(1-\frac{\alpha_{1} \gamma}{1+\alpha_{1}} \frac{\varepsilon^{3}}{4}\right) \sin \theta+\alpha_{1} \gamma \frac{1+\alpha_{2}}{1+\alpha_{1}} \frac{9 \varepsilon^{4}}{32} \sin 2 \theta+. .
$$

Comparison with Eq. (3) reveals that the deformation of the heat flow due to the presence of $B$ influences the quasislip velocity in two ways: The expression in parentheses changes the effective value of the term proportional to $\sin \theta$, whereas the remainder adds higher Fourier componenents $\sin n \theta$. Both corrections depend on the parameter $\varepsilon$ and thus in principle have to be added to the perturbation series of Sect. 3 .

The prefactor of $\sin \theta$ in (30) appear in the coefficients $p_{1}$ and $q_{3}$ defined in (7), and in those related through (10). As a consequence, their series are modified at the order $\varepsilon^{3}$. On the other hand, the Fourier components $\sin n \theta$ add new source fields in Stokes' equation and thus result in additional terms in (6). For example, the above prefactor of $\sin 2 \theta$ appears in 
the coefficients $p_{2}$ and $q_{4}$. Since these corrections are of the order $\varepsilon^{n+2}$, they contribute to the third and higher reflections only; the analytic results of Eqs. (13)-(18) are of order $\varepsilon^{2}$ and thus remain unchanged.

Fig. 3 indicates that the series expansion of Sect. 3 in powers of $\varepsilon$ converges rapidly, and that third and higher orders contribute rather little. This picture remains valid when accounting for the conductivity mismatch. The corrections arising from (30) are in general smaller than those of Sect. 3. Note that the $\alpha_{1}$ and $\gamma$ are strictly smaller than unity, and that the numerical prefactor of $\varepsilon^{3}$ in (30) is smaller than in (16).

According to (24), the corrections in (30) are proportional to the heat conductivity mismatch both at the particle surface and at the solid boundary; they vanish if $\kappa$ is continuous at one of the interfaces, $\kappa_{L}=\kappa_{P}$ or $\kappa_{L}=\kappa_{B}$. The most significant effect arises if the conductivities of both the particle and the solid boundary are much larger than that of the liquid. In the experiment of Ref. [16], the conductivity of the particles $\left(\kappa_{P}=1 \mathrm{~W} / \mathrm{Km}\right.$ for silica and $0.1 \mathrm{~W} / \mathrm{Km}$ for polystyrene) is not very different from that of water $\left(\kappa_{L}=0.6\right.$ $\mathrm{W} / \mathrm{Km})$; both are much smaller than that of the sapphire support $\left(\kappa_{B}=35 \mathrm{~W} / \mathrm{Km}\right)$. In Ref. [17] the particles and the solid boundary are of glass, with a heat conductivity about twice as large as the solvent. Inserting these numbers in (30) leads to rather insignificant corrections.

The discussion of the deformation of a temperature gradient in. (20)-(30) is readily adapted to that of an applied electric field $E$, by replacing $\nabla T$ with $E$ and the heat conductivities $\kappa_{i}$ with the corresponding permittivities $\epsilon_{i}$. In the case of a perfectly conducting solid boundary, the interface $S$ is an equipotential surface, or isotherm, and results in $\gamma=1$.

\section{SUMMARY}

In this paper we have studied the fluid velocity field in the vicinity of a colloidal particle with interfacial forces, that is immobilized by a solid boundary $S$. By iterating reflections at $S$ and at the particle surface, we obtained the fluid flow as a power series in the parameter $\varepsilon$. For particles at large distance from the wall $(\varepsilon \ll 1)$ the wall hardly affects the flow pattern. We summarize our main results for large $\varepsilon$.

(i) Beyond the usual one-reflection approximation, which corresponds to $k=0$ in our notation, we find qualitatively different corrections in linear and quadratic order in $\varepsilon$. For 
particles close to the confining wall, $\varepsilon \sim 1$, the linear term enhances the velocity field by a factor of about 2, yet leaves the overall flow pattern unchanged.

(ii) In quadratic order $k=2$, novel terms arise that modify the fluid velocity in the immediate vicinity of the bead, but vanish rapidly beyond one or two particle radii.

(iii) As a consequence, the fit of experimental data in Fig; 4 is sensitive to linear corrections only. The quasislip velocity resulting from $k=1$ is half as large as that obtained at zeroth order. Terms of second and higher order are rather insignficant.

(iv) The presence of the solid boundary locally deforms the external field which, as a consequence, depends itself on the parameter $\varepsilon$. Finite corrections appear at third order; though they have to be considered in a formal expansion, their contributons are of no consequence for the above conclusions.

[1] J.L. Anderson, Annu. Rev. Fluid Mech 21, 61 (1989)

[2] S. Duhr, D. Braun, Phys. Rev. Lett. 97, 038103 (2006)

[3] B. Abécassis et al., Nature Materials, 7785 (2008)

[4] H.-R. Jiang et al., Phys. Rev. Lett. 102, 208301 (2009)

[5] H. Brenner, Chem. Eng. Sci. 16, 242 (1961)

[6] J. Happel, H. Brenner, Low-Reynolds Number Hydrodynamics, Kluwer Academic Publisher (1991)

[7] H.J. Keh, J.L. Anderson, J. Fluid Mech. 153, 417 (1985)

[8] T.M. Squires, M.P. Brenner, Phys. Rev. Lett. 85, 4976 (2000)

[9] T.M. Squires, J. Fluid Mech. 443, 403 (2001)

[10] M. Giersig, P. Mulvaney, Langmuir 9, 3408-3413 (1993)

[11] M. Böhmer, Langmuir 12, 5747 (1996)

[12] M. Trau, D. Saville, A.I. Askay, Science 272, 706 (1996).

[13] S-R. Yeh et al., Nature 386, 57 (1997)

[14] W.D. Ristenpart et al., Phys. Rev. E 69, 021405 (2004)

[15] Y. Solomentsev, M. Böhmer and J.L. Anderson, Langmuir 13, 6058 (1997).

[16] F.M. Weinert, D. Braun, Phys. Rev. Lett. 101, 168301 (2008)

[17] R. Di Leonardo, F. Ianni, G. Ruocco, Langmuir 25, 4247 (2009) 
[18] E. Ruckenstein, J. Coll. Interf. Sci. 83, 77 (1981)

[19] R. Piazza, A. Parola, J. Phys. Cond. Matt. 20, 153102 (2008)

[20] A. Würger, Phys. Rev. Lett. 101, 108302 (2008)

[21] J.R. Blake, Proc. Camb. Phil. Soc. 70, 303 (1971)

[22] P. Epstein, Z. Phys. 54, 537 (1929)

[23] J. Santana-Solano, D.T. Wu, D.W.M. Marr, Langmuir 22, 5932 (2006). 